1720 patients were found to be subject to an advisory. Of these patients, 1234 were software advisories and 486 mechanical advisories.

This resulted in 1237 patients requiring device follow up to be intensified.

One hour of additional support was calculated per patient advisory. This was made up of 20 minutes for the initial interrogation and 40 minutes for extra administration and subsequent follow up if required. An average of 1720 hours was spent on patient advisories over a 10 -year period resulting in one extra clinic session per week.

Conclusion We calculated that $33 \%$ of all patients seen in clinic in the last 10 years have been subject to an advisory. On average over the ten year period this has required an additional 1720 hours per patient advisory resulting in an extra whole physiology clinic session per week. This represents a significant challenge to the Pacing workforce.

Our data shows that there are more software related advisories than hardware advisories. Software solutions resolve the issue but are often delayed.

Device advisories have now become a major complication in pacemaker implantation with a significantly higher frequency than procedural complications. One could consider adding this finding to the discussion with the patient prior to device implant.

Conflict of Interest None at time of writing

\section{A META-ANALYSIS ON THE IMPACT OF CONTACT FORCE SENSING CATHETERS ON OUTCOMES IN ATRIAL FIBRILLATION ABLATION: A FOCUS ON STUDY DESIGN}

${ }^{1}$ Amit Kaura*, ${ }^{2}$ Nicholas Sunderland, ${ }^{2}$ Francis Murgatroyd, ${ }^{2}$ Para Dhillon, ${ }^{2}$ Paul Scott. ${ }^{1}$ NIHR Imperial College London Biomedical Research Centre and Imperial College Healthcare NHS Trust; ${ }^{2}$ King's College Hospital NHS Foundation Trust

10.1136/heartjnl-2019-BCS.27

Background One determinant of failure of atrial fibrillation (AF) ablation is the lack of transmural lesions. Contact force sensing (CFS) catheters enable real-time assessment of the amount of force applied at the catheter-tissue interface, with higher contact force associated with lesions of greater size. Previous meta-analyses have pooled results from randomized controlled trials (RCTs) and observational studies (OSs) and concluded that CFS catheters improve clinical outcomes. This meta-analysis sought to compare the efficacy and safety of CFS catheters with non-CFS catheters for AF radiofrequency ablation.

Methods A total of 28 studies were identified using Embase and Medline databases; 8 RCTs and 20 OSs. For the primary outcome of atrial tachyarrhythmia recurrence, data from RCTs and OSs were analysed according to study design. Secondary outcomes included ablation time and all procedure-related complications. Summary estimates for each variable were calculated using the random-effects model based on DerSimonian and Laird's meta-analytic statistical method.

Results In the 8 RCTs there were a total of 844 patients (419 in CF group and 425 in non-CF group). In the 20 OS there were 3900 patients (1523 in CF group and 2377 in non-CF group). The median age of patients was 60.3 years (59.0 years in RCTs and 60.7 years in OSs). The majority were male (74.0\% in RCTs and $69.6 \%$ in OSs). The median follow-up time post-AF ablation was 12 months in both RCTs and OSs.
While CFS did not lead to a significant reduction in atrial tachyarrhythmia recurrence in RCTs $(n=844$, risk ratio (RR) $0.98,95 \%$ confidence interval (CI) 0.80 to $1.19, \mathrm{p}=0.81$ ), a significant reduction was observed in OSs $(n=2,259$, RR 0.74 , $95 \%$ CI 0.65 to $0.84, \mathrm{p}<0.0001$ ), both at a median follow-up of 12 months.

Data on ablation time were available for 6 RCTs $(n=751)$ and 14 OSs $(n=3,152)$. The use of CFS catheters had no significant impact on ablation time in both RCTs (-0.36 minutes, 95\% CI -3.32 to $2.59, \mathrm{p}=0.81)$ and OSs ( -3.68 minutes, $95 \%$ CI -7.91 to $0.55, \mathrm{p}=0.09)$.

For both RCTs $(n=706)$ and OSs $(n=3,427)$, the incidence of all procedure-related complications was similar in the CFS and non-CFS groups (RCTs, RR 0.99, 95\% CI 0.55 to 1.78 , $\mathrm{p}=0.97$; OSs, RR 0.78 , 95\% CI 0.53 to $1.16, \mathrm{p}=0.22$ ).

Conclusion Although OSs have demonstrated positive results, data from RCTs have failed to show any significant benefit from CFS catheters in terms of procedural success in AF ablation. The safety profile of CFS catheters was similar to nonCFS catheters in both RCTs and OSs.

Conflict of Interest No conflicts on interest

\section{THE SHORT PR INTERVAL IN YOUNG ATHLETES}

${ }^{1} \mathrm{G}$ Parry-Williams*, ${ }^{1} \mathrm{~A}$ Malhotra, ${ }^{2} \mathrm{H}$ Dhutia, ${ }^{3} \mathrm{~A}$ Cajucom, ${ }^{1} \mathrm{~J}$ Basu, ${ }^{1} \mathrm{C}$ Miles, ${ }^{1} \mathrm{M}$ Papadakis, ${ }^{1} S$ Sharma* ${ }^{1}$ St George's University of London, Molecular and Clinical Sciences Research, London, United Kingdom ${ }^{2}$ University Hospitals of Leicester NHS Trust, Cardiology, Leicester, United Kingdom ${ }^{3}$ St George's University of London, London, United Kingdom.

\subsection{6/heartjnl-2019-BCS.28}

Background International recommendations for interpreting the athlete's ECG define a short PR interval as $<120 \mathrm{~ms}$. Despite the recommendation that asymptomatic athletes do not require further investigation unless an accessory pathway is suspected, athletes are not infrequently referred for further evaluation. The prevalence of a short PR in athletes has not previously been reported.

Purpose To investigate the prevalence of short PR in young athletes and its association with age, gender and ethnicity.

Methods Between 2011-2014, 15,572 athletes aged 14-35 underwent cardiac screening including an ECG. An athlete was defined as an individual participating in sport $6 \mathrm{hrs} / \mathrm{wk}$. ECGs were analysed by 2 independent experts. Athletes in whom the PR interval was not fixed were excluded. A short PR was defined as $<120 \mathrm{~ms}$.

Results Amongst 15,572 athletes (mean age 18.6 years, 92\% white, $80 \%$ male), the mean PR interval was $151 \mathrm{msec}$ and shorter in females vs. males, white vs. non-white and adolescent ( $\leq 16$ years) vs. older (17-35 years) athletes (table 1).

An isolated short PR was present in 765 (4.9\%) athletes and was more common in females vs. males $(6.2 \%$ vs. $4.2 \%$; $\mathrm{p}<0.0001$ ) (figure 1). The prevalence of short PR in athletes reduced significantly with advancing age, present in $9 \%$ of 14 year olds but only $3.2 \%$ of $17-35$ year olds $(p<0.0001)$. The prevalence of short PR was similar between ethnic groups $(4.7 \%$ white vs $4.2 \%$ non-white; $\mathrm{p}=0.55)$. The overall prevalence of the Wolff-Parkinson-White pattern was $0.08 \%$.

Conclusions A short PR interval is a fairly frequent finding in this cohort with a predilection for younger and female athletes. Possible explanations for shorter conduction time include anatomically smaller hearts, higher sympathetic tone or 